\title{
PREVALÊNCIA DE FATORES PSICOLÓGICOS EM PACIENTES COM DISFUNÇÃO TEMPOROMANDIBULAR ATENDIDOS NA CLÍNICA ODONTOLÓGICA DA UNIVERSIDADE ESTADUAL DE FEIRA DE SANTANA.
}

Caroline Oliveira dos Santos Freitas ${ }^{1}$; Franco Arsati²; Valéria Souza Freitas ${ }^{3}$

1. Bolsista PIBIC/CNPq, Graduanda em Odontologia, Universidade Estadual de Feira de Santana, e-mail: carolfreitasodonto@gmail.com

2. Orientador, Departamento de Ciências Biológicas, Universidade Estadual de Feira de Santana, e-mail:farsati@gmail.com

3. Pesquisadora do Núcleo de Câncer Oral, Departamento de Saúde, Universidade Estadual de Feira de Santana, e-mail: valeria.souza.freitas@gmail.com

PALAVRAS-CHAVE: fatores psicológicos; disfunção temporomandibular, dor.

\section{INTRODUÇÃO}

A Disfunção temporomandibular (DTM) é um termo que engloba uma série de disfunções e desordens que afetam a (ATM) e/ ou os músculos mastigatórios. Os sinais e sintomas são variáveis, no entanto, os três principais são dor, limitação da abertura de boca, e sons na articulação temporomandibular. A dor é geralmente a queixa principal (LERESCHE, 1997). A DTM e dor orofacial acometem preferencialmente mulheres em idade reprodutiva (BOVE; GUIMARÃES; SMITH, 2005). Ainda não foi identificada uma causa isolada e universal para explicar o desenvolvimento da DTM, de forma que a sua etiologia é considerada multifatorial envolvendo fatores físicos e psicológicos (Carrara et al., 2010). Os fatores psicológicos envolvidos nas desordens temporomandibulares estão sendo cada vez mais estudados e discutidos e podem ser divididos em emocionais (ansiedade, depressão e estresse) e fatores cognitivos (expectativas e significados quanto à sensação de dor (CESTARI; CAMPARIS, 2002). Tem sido observado que pessoas expostas a fatores estressantes comumente demonstram um aumento de hiperatividade do músculo masseter (CESTARI; CAMPARIS, 2002). Em outros estudos já foi demonstrado que a depressão e a catastrofização da dor contribuem para o aparecimento e persistência de DTM (VELLY; LOOK; ; SCHIFFMAN , 2010;VELLY; LOOK; CARLSON, 2011). Indivíduos com dor relacionada à DTM apresentam maiores níveis de estresse, ansiedade, depressão, somatização e pensamentos catastróficos em comparação a indivíduos saudáveis (MACFARLANE; KENEALY; KINGDON, 2009; (DE LEEUW et al., 2005; CARLSON, 2007; MANFREDINI; LANDI; BANDETTINI DI POGGIO A, 2003; VISSCHER; OHRBACH; VAN WIJK, 2010). Diante do exposto, o objetivo do presente trabalho foi avaliar a prevalência de fatores psicológicos em pacientes com disfunção temporomandibular atendidos na clínica odontológica da UEFS, no período de agosto de 2016 a agosto de 2017.

\section{METODOLOGIA}

\section{Caracterização do estudo}

Trata-se de um estudo observacional do tipo descritivo.

\section{População do estudo}

Participaram deste estudo todos os pacientes diagnosticados com disfunção temporomandibular (DTM) e atendidos na clínica odontológica da Universidade Estadual de Feira de Santana (UEFS- BA), no período de agosto de 2016 a agosto de 2017.

a) Critérios de inclusão: Pacientes com DTM atendidos na clínica odontológica da UEFS, com no mínimo 18 anos de idade e que assinaram o termo de consentimento livre e esclarecido.

b) Critérios de exclusão: Foram excluídos os pacientes diagnosticados com dores orofaciais não relacionadas às DTMs, e com histórico recente de cirurgia na ATM e portadores de doença mental 


\section{Coleta de dados}

A coleta de dados foi realizada durante a consulta inicial dos pacientes. Inicialmente os pacientes foram submetidos a uma anamnese e exame físico segundo o protocolo do $R D C / T M D$ (Schiffman et al., 2010) e, na sequência, preencheram escalas analógicas visuais numéricas da dor (dor atual; a média da dor nos últimos 30 dias e a pior dor nos últimos 30 dias) (Pardo et al., 2006), a escala de estresse percebido (Cohen et al., 1983), a escala hospitalar de ansiedade e depressão (HERRMANN, 1997) e a escala de catastrofização de dor (LOPES et al., 2015).

\section{Análise de dados}

Para a elaboração do banco, tabulação de dados e para análise descritiva foi utilizado o programa BioEstat 5.0 (Instituto de Desenvolvimento Sustentável Mamirauá, Tefé, AM, Brasil). Os dados coletados foram submetidos à análise descritiva, a fim de apresentar a distribuição de cada variável na população examinada. Em acréscimo, as varáveis estudadas foram submetidas ao teste de correlação de Pearson. Foi adotado o nível de significância de $5 \%$.

\section{RESULTADOS E DISCUSSÃO}

A população constitui-se de 4 homens de 26 mulheres e uma média de idade de 36,28 anos $\pm 10,86$ (média \pm desvio-padrão), evidenciando uma predileção por mulheres em idade reprodutiva, como já citada em demais estudos na literatura, que mencionam ainda que há um possível papel etiológico para os hormônios reprodutivos femininos nessas condições de dor (LERESCHE, 1997). A sensação dolorosa do paciente no momento da consulta $(2 ; 4,75$ mediana; intervalo interquartílico), a média de dor sentida pelo paciente nos últimos 30 dias $(5 ; 5,4)$ e a pior dor experimentada nos últimos 30 dias $(7,5 ; 3)$ estão demonstradas no gráfíco de caixas (Gráfico 1). Metade dos pacientes foram classificados como estressados (Tabela 1) (COHEN et al., 1983). Foi observado ainda, uma correlação positiva, porém fraca, $(p=0,021$; $\mathrm{r}=0,44$ ) entre o estresse e a média de dor relatada pelo paciente nos últimos 30 dias. Estudos longitudinais observaram adultos saudáveis sem nenhum histórico prévio de DTM, e concluíram que aqueles indivíduos com níveis aumentados de estresse percebido foram justamente os que apresentaram a maior probabilidade de desenvolvimento de DTM, em comparação a indivíduos com menores níveis de estresse percebido (SLADE et al., 2007; FILLINGIM et al., 2014). Os sintomas de ansiedade, depressão e catastrofização estão demonstrados na tabela 1. No presente trabalho, os sintomas de ansiedade e depressão, divididos por categorias (sem sintoma, leve, moderada e severa), somaram a frequência de 50 e 53,4\%, respectivamente, valores muito aquém dos observados por Bonjardim et al., 2005), os quais constataram em uma amostra de adolescentes com sinais e sintomas de DTM, ansiedade e depressão em somente 17 e $28 \%$ dos indivíduos. Os sintomas de ansiedade do presente trabalho, mas não os de depressão, assemelham-se aos apresentados por Costa et al. (2015), que observaram o efeito do tratamento de pacientes com DTM sobre a redução desses fatores psicológicos. Antes do tratamento, 50\% e 33,2\% de seus pacientes apresentaram sintomas de ansiedade e depressão. Os sintomas mais altos de depressão na presente amostra podem estar relacionados com o menor nível socioeconômico e menor qualidade de vida dos pacientes atendidos na clínica de Odontologia da UEFS. A catastrofização da dor pode ser definida como o conjunto de pensamentos negativos e exagerados centrados na sensação de dor e de incapacidade de lidar com ela. Dentre os fatores psicológicos, a catastrofização da dor é um dos maiores preditores da intensidade da dor, estresse e insucesso do tratamento em pacientes com dor crônica (Costa et al., 2015). Nosso estudo revelou que 36,66\% dos portadores de DTM são acometidos pela catastrofização, e esta correlacionou-se positivamente com a "dor atual" ( $\mathrm{p}=0.017 ; \mathrm{r}=0,43)$, com a média de dor nos últimos 30 dias ( $\mathrm{p}<$ $0.0001 ; \mathrm{r}=0,67)$ e com a pior dor experimentada nos últimos 30 dias $(\mathrm{p}<0.0011 ; \mathrm{r}=0,56)($ Gráfico 2). 
Tabela 1: Distribuições absolutas (n) e relativas (\%) dos fatores psicológicos dos pacientes com DTM atendidos na clínica Odontológica de UEFS no período de agosto de 2016 a agosto de 2017.

\begin{tabular}{|c|c|}
\hline & Pacientes $(n=30)$ \\
\hline \multicolumn{2}{|l|}{ Estresse, $\mathrm{n}(\%)$} \\
\hline Sem estresse & $15(50 \%)$ \\
\hline Com estresse & $12(40 \%)$ \\
\hline Dados perdidos & $3(10 \%)$ \\
\hline \multicolumn{2}{|l|}{ Ansiedade, n (\%) } \\
\hline Sem ansiedade & $15(50 \%)$ \\
\hline leve & $6(20 \%)$ \\
\hline moderada & $6(20 \%)$ \\
\hline Severa & $3(10 \%)$ \\
\hline Com ansiedade* & $15(50 \%)$ \\
\hline \multicolumn{2}{|l|}{ Depressão, $n(\%)$} \\
\hline Sem depressão & $14(46,6 \%)$ \\
\hline Leve & $11(36,6 \%)$ \\
\hline Moderada & $4(13,3 \%)$ \\
\hline Severa & $1(3,5 \%)$ \\
\hline Com depressão* & $16(53,4 \%)$ \\
\hline \multicolumn{2}{|l|}{ Catastrofização $n(\%)$} \\
\hline Sem catastrofização & $19(63,4 \%)$ \\
\hline Com catastrofização & $11(36,6 \%)$ \\
\hline
\end{tabular}

*soma das categorias leve, moderada e severa

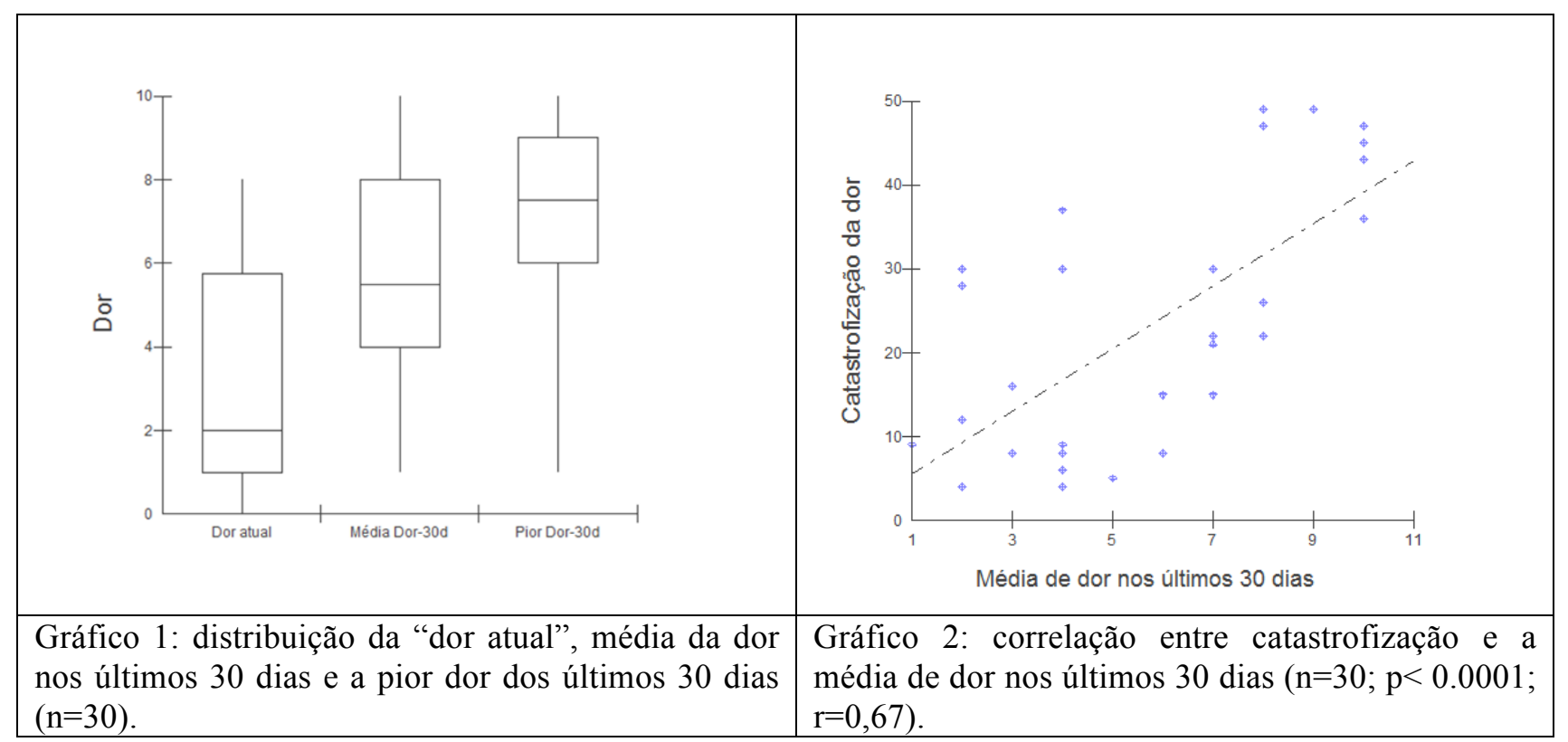

\section{Conclusão}

Os resultados do presente trabalho demonstraram que, nos pacientes com disfunção temporomandibular atendidos na clínica Odontológica da UEFS entre agosto de 2017 e agosto de 2018, a prevalência de sintomas de estresse, ansiedade e depressão foram altos, e que, o estresse psicológico e, principalmente, a catastrofização da dor estão associados com a maior intensidade de dor em pacientes com disfunção temporomandibular. 


\section{REFERÊNCIAS}

AMANTEA DV, NOVAES AP, CAMPOLONGO GS, B. T. A importância da avaliação postural no paciente com disfunção temporomandibular. Acta Ortop. Acta Ortop. Bras, v. 12, n. 3, p. 155-9, 2004.

ARNTZ A, HILDEBRAND M, V. D. H. M. Overprediction of anxiety, and disconfirmatory processes, in anxiety disorders. Behav Res Ther, v. 32, p. 709-22, 1994.

BOVE, S. R. K.; GUIMARÃES, A. S.; SMITH, R. L. Caracterização Dos Pacientes De Um Ambulatório De Disfunção Temporomandibular E Dor Orofacial. Rev Latino-am de enfermagem, v. 13, n. 5, p. 686-691, 2005.

Bonjardim LR, Gavião MB, Pereira LJ, Castelo PM. Anxiety and depression in adolescents and their relationship with signs and symptoms of temporomandibular disorders. Int J Prosthodont. 2005 JulAug;18(4):347-52.

CA, Q. Classificação e tratamento das disfunções temporomandibulares: qual o papel do fonoaudiólogo no tratamento dessas disfunções? Rev CEFAC, v. 2, n. 2, p. 15-22, 2000. CARLSON, C. R. Psychological Factors Associated with Orofacial Pains. Dental Clinics of North America, v. 51, n. 1, p. 145-160, 2007.

CESTARI, K.; CAMPARIS, C. M. Fatores Psicológicos : sua Importância no Diagnóstico das Desordens Temporomandibulares. Jornal Brasileiro de Oclusão, ATM e Dor Orofacial, v. 2, n. 5, p. 54-60, 2002.

Cohen S, Kamarck T, Mermelstein R. A global measure of perceived stress.J Health Soc Behav. 1983 Dec;24(4):385-96.

DE LEEUW R, BERTOLI E, SCHMIDT JE, ET AL. Prevalence of traumatic stressors in patients with temporomandibular disorders. Maxillofac Surg 2005, v. 63, p. 42-50, 2005.

Herrmann C. International experiences with the Hospital Anxiety and Depression Scale--a review of validation data and clinical results. J Psychosom Res. 1997 Jan;42(1):17-41.

LeResche L. Epidemiology of temporomandibular disorders: Implications for the investigation of etiologic factors. Crit Rev Oral Biol Med, p. 291-305, 1997.

Leeuw R de, Klasser GD. Orofacial Pain: Guidelines for Assessment, Diagnosis, and Management, Fifth Edition. Chicago : Quintessence Publishing Co, Inc. 2013.

Lopes RA, Dias RC, Queiroz BZ, Rosa NM, Pereira Lde S, Dias JM, Magalhães Lde C.Psychometric properties of the Brazilian version of the Pain Catastrophizing Scale for acute low back pain.Arq Neuropsiquiatr. 2015 May;73(5):436-45.

MACFARLANE TV, KENEALY P, KINGDON HA, ET AL. Orofacial pain in young adults and associated childhood and adult- hood factors: Results of the population study, Wales, United Kingdom. Commun Dent Oral Epidemiol, v. 37, p. 438-450, 2009.

MANFREDINI D, LANDI N, BANDETTINI DI POGGIO A, ET AL. A critical review on the importance of psychological fac- tors in temporomandibular disorders. Minerva Stomatol, v. 52, p. 321-326, 2003.

Pardo C, Muñoz T, Chamorro C; Analgesia and Sedation Work Group of SEMICYUC Med Intensiva. Monitoring pain: recommendations of the Analgesia and Sedation Work Group of SEMICYUC].2006 Nov;30(8):379-85.

Schiffman EL, Ohrbach R, Truelove EL, et al. The Research Diagnostic Criteria for Temporomandibular Disorders. V: methods used to establish and validate revised Axis I diagnostic algorithms. J Orofac Pain 2010;24(1):63-78.

VELLY AM, LOOK JO, CARLSON C, ET AL. The effect of catastrophizing and depression on chronic pain - a prospective cohort study of temporomandibular muscle and joint pain disorders. Pain, v. 152, p. 2377-2383, 2011.

VELLY AM, LOOK JO, SCHIFFMAN E, ET AL. The effect of fibromyalgia and widespread pain on the clinically sig- nificant temporomandibular muscle and joint pain disorders - a prospective 18month cohort study. J Pain, v. 11, p. 1155-1164, 2010.

VISSCHER CM, OHRBACH R, VAN WIJK AJ, ET AL. The Tampa Scale for Kinesiophobia for temporomandibular dis- orders (TSK-TMD). Pain, v. 150, p. 492-500, 2010. 